\title{
Assistive Teleoperation for Manipulation Tasks
}

\author{
Anca D. Dragan \\ The Robotics Institute, Carnegie Mellon \\ adragan@cs.cmu.edu
}

Categories and Subject Descriptors: I.2.9 Artificial Intelligence: Robotics

Keywords: assistive robotics, human-robot interaction, teleoperation

\section{INTRODUCTION}

How should a human user and a robot collaborate during teleoperation? The user understands the full semantics of the task: they know, for example, what the robot should search for in a cupboard, or that it should be more careful when moving near a glass of water than near a box of tissues. Since the robot might not have this knowledge, allowing it to operate fully autonomously may be risky; its model is incomplete and its policy might be wrong. On the other hand, teleoperating the robot through every motion is slow and tiresome, especially on difficult tasks. Between these two extremes lies a spectrum, from almost no assistance at all (very timid) to full autonomy (very aggressive). So what is the appropriate level of assistance? And how do factors like task difficulty and policy correctness affect this decision?

We address these question by formalizing assistance as policy blending, where user input $U$ and robot policy $P$ are blended via a state-dependent arbitration function $\alpha$ :

\begin{tabular}{|c|c|c|}
\hline User Input & & \\
\hline$U$ & \multirow{2}{*}{$\begin{array}{l}\text { Policy Blending } \\
(1-\alpha) U+\alpha P\end{array}$} & \multirow{2}{*}{ Robot Action } \\
\hline Robot Policy & & \\
\hline
\end{tabular}

Modifying $\alpha$ enables us to manipulate how aggressively the robot assists. For example, $\alpha=0$ everywhere corresponds to no assistance, and $\alpha=1$ everywhere corresponds to full autonomy. The two $\alpha$ functions from Fig. 1(c) represent a timid and an aggressive mode in between these extremes. To explore the effects of $\alpha$, we performed a study where users teleoperated our robot HERB to grasp objects from a table, with varying assistance from HERB's own policy for grasping the objects. We manipulated three factors: aggressiveness of assistance, difficulty of task, and correctness of robot policy, and measured the time to success and user preference. All users favored aggressive assistance on hard tasks, when the robot's policy was correct. In contrast, they clearly preferred timid assistance on tasks where the robot's policy was wrong. Opinions were split on easy tasks, depending on how much they wanted to remain in control of the robot. Overall, although aggressive assistance is more efficient when the policy is right, robots might make mistakes that are much easier to handle with timid assistance. This emphasizes the importance of correctness, of trust, and of legibility of motion in order for teleoperation to efficiently transfer the load from the human onto the robot.

Copyright is held by the author/owner(s).

HRI'12, March 5-8, 2012, Boston, Massachusetts, USA.

ACM 978-1-4503-1063-5/12/03.

\author{
Siddhartha S. Srinivasa \\ The Robotics Institute, Carnegie Mellon \\ siddh@cs.cmu.edu
}

\section{PRIOR WORK}

Policy blending provides a unified view of much prior work in assistive teleoperation, as different behaviors that have been proposed may be cast as different arbitration functions. For example, in some methods the robot always has full control of some local aspect of the motion ( $\alpha$ is always 1$)$ [2]. In others, the robot takes control at a certain trigger point, depending on proximity to the goal [4], or to a preset path [5]. There are also instances of more timid assistance, in which the robot never fully takes control. In [1], the intensity of assistance increases with the probability of the predicted user intent, but never past a maximum value.

Studies comparing modes of assistive teleoperation obtained seemingly contradictory results. Although You and Hauser [6] found aggressive assistance better, Kim et al. [3] showed that users preferred a manual mode. Our analysis indicates that the variance in task difficulty between the studies (shown in the timing difference between the timid and aggressive modes) explains the two findings and makes the results consistent.

\section{EXPERIMENTAL DESIGN}

We tasked users with teleoperating HERB to grasp an object from a table. We implemented a whole-body interface that tracks their skeleton (OpenNI, www.openni.org), yielding the user input $U$ (Fig. 1 (a)). The arbitration function $\alpha$ blends $U$ with a policy $P$.

\subsection{Manipulation of the three factors}

We instructed users to reach for the farther of two objects. We manipulated policy correctness by changing $P$ : in the right case, $P$ moves directly towards the target object; in the wrong case, $P$ moves towards the object closest to the user's configuration (initially the wrong one). Fig. 1 (b) shows examples of the two cases in green and red, respectively. We manipulated task difficulty by changing the target object's location. Fig. 1 (b) shows an example of an easy (grasp the bottle) vs. a hard task (grasp the box). These two factors lead to 4 tasks: Easy-Right, Easy-Wrong, Hard-Right and Hard-Wrong.

We manipulated aggressiveness by modifying the arbitration function (Fig. 1 (c)). This function depends on a measure of the robot's confidence $c$ in the predicted policy. For simplicity, we chose an intuitive definition of confidence: $c$ increases as the distance $d$ from the user's configuration to the policy's target decreases, $c=\max \left(0,1-\frac{d}{D}\right)$. The timid mode increases the assistance with the confidence and plateaus at a max. value, never taking control away from the user. The aggressive mode takes control from the user as soon as the confidence increases past a min. value.

\subsection{Subject allocation}

To maximize the number of data points per condition, we chose a within-subjects design. Each of our 8 participants (all students, 4 males and 4 females) executed both modes 


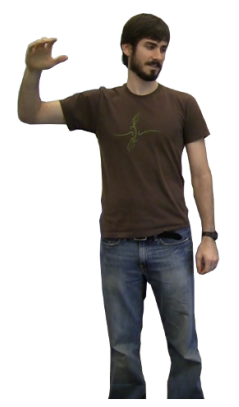

(a)

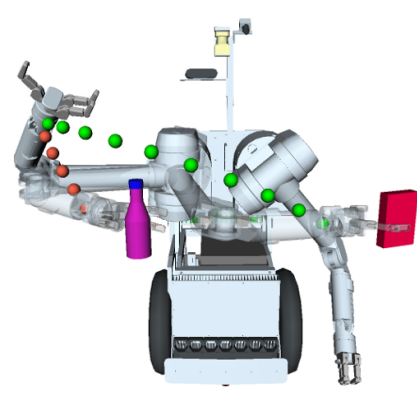

(b)

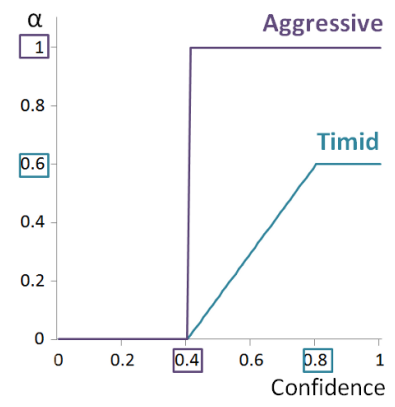

(c)

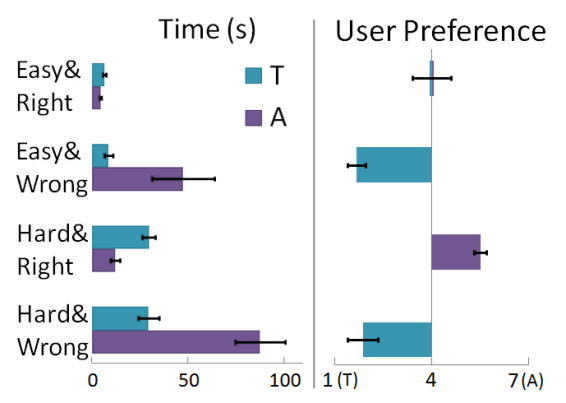

(d)

Figure 1: a) The user's arm pose serves as the input $H$. b) The robot chooses one of the two policies (one to each possible target) and blends it with the user input. c) The arbitration functions for timid and aggressive assistance, depending on the confidence in the policy. d) A comparison of the two modes on each task.

on each task. To avoid ordering effects, we used a balanced Latin square for the task order, and balanced the order of the modes within each task.

\subsection{Dependent variables and covariates}

We measured the amount of time (performance) per condition. We expected this to correlate to the user's preference. We identified the following confounds: the users' initial teleoperation skill, their rating of the robot without assistance, and the learning effect. To control for these, users went though a training phase, teleoperating the robot without assistance. This partially eliminated the learning effect and gave us a baseline for their timing and ratings. We used these as covariates, together with number of tasks completed at any point (a measure of in-task practice).

\subsection{Hypotheses}

1. All factors have a significant effect on task performance.

2. The aggressive mode performs better on Hard-Right tasks, while the timid mode performs better on EasyWrong tasks.

We leave as open questions what happens with both complications (Hard-Wrong), or with neither (Easy-Right).

\section{RESULTS}

\subsection{Performance Analysis}

The average time per task was approximately 28s. We performed a factorial repeated-measures ANOVA with Bonferroni corrections for multiple comparisons and a significance threshold of $\mathrm{p}=0.05$, which resulted in a good fit of the data $\left(R^{2}=0.66\right)$. In line with our first hypothesis, we found main effects for all three factors: hard tasks took $22.9 \mathrm{~s}$ longer than easy ones $(\mathrm{F}(1,53)=18.45, \mathrm{p}<.001)$, tasks where the policy was wrong took $30.1 \mathrm{~s}$ longer than when right $(\mathrm{F}(1,53)=31.88, \mathrm{p}<.001)$, and the aggressive mode took overall 19.4 s longer than the timid $(\mathrm{F}(1,53)=13.2, \mathrm{p}=.001)$. We found a significant interaction effect between aggressiveness and correctness, showing that when wrong, being timid is significantly better than being aggressive. This is confirmed in Fig. 1 (d), which compares the means and standard errors on each task: the timid mode is better both on EasyWrong and Hard-Wrong. The same plot also shows that the timid mode performed about the same on Easy-Right, and, as expected, worse on Hard-Right (the aggressive is lower than the timid for every user). Surprisingly, the interaction effect among all factors was only marginally significant $(\mathrm{F}(1,53)=2.63, \mathrm{p}=.11)$. We believe that increasing the size of our user pool will strengthen this effect.

\subsection{Preference Analysis}

Fig 1 (d) also shows the user's preferences on each task, which are in line with the timing results. The two measures were indeed correlated (Pearson's $r(30)=.66, \mathrm{p}<.001$ ). The outliers were users with stronger preferences than the time difference would indicate. For example, some users strongly preferred the timid mode on Hard-Wrong tasks, despite the time difference not being as high as with other users. The opposite happened on Hard-Right tasks, on which some users strongly preferred the aggressive mode despite a small time difference, commenting that they appreciated the precision of the autonomy. On Easy-Right tasks, the opinions were split and some users preferred the timid mode despite a slightly longer time, motivating that they felt more in control of the robot. Overall, although performance difference is a good indicator of the preference, it does not capture a user's experience in its entirety.

\section{CONCLUSION}

We identified aggressiveness as a key aspect of assistance and showed that task difficulty and policy correctness influence the mode of assistance. We found that being wrong, yet aggressive has the largest negative effect, and that user preferences were sometimes more drastic than the performance difference would indicate. Users also commented that after seeing the robot be wrong, they did not trust the aggressive mode, and were unable to tell from its motion whether it was right. This suggests that even though autonomy makes teleoperation easier, issues of correctness, trust, and behavior legibility must be addressed for effective assistance.

\section{REFERENCES}

[1] D. Aarno, S. Ekvall, and D. Kragic. Adaptive virtual fixtures for machine-assisted teleoperation tasks. In ICRA, 2005.

[2] T. Debus, J. Stoll, R. Howe, and P. Dupont. Cooperative human and machine perception in teleoperated assembly. In $I S E R, 2000$.

[3] D. Kim, R. Hazlett-Knudsen, H. Culver-Godfrey, G. Rucks, T. Cunningham, D. Porteande, J. Bricout, Z. Wang, and A. Behal. How autonomy impacts performance and satisfaction. IEEE Trans. on Systems, Man and Cybernetics, $\mathrm{PP}(99): 1-13$, 2011.

[4] J. Kofman, X. Wu, T. Luu, and S. Verma. Teleoperation of a robot manipulator using a vision-based human-robot interface. IEEE Trans. on Industrial Electronics, 52(5):1206 - 1219, 2005.

[5] M. Li and A. Okamura. Recognition of operator motions for real-time assistance using virtual fixtures. In HAPTICS, 2003.

[6] E. You and K. Hauser. Assisted teleoperation strategies for aggressively controlling a robot arm with $2 \mathrm{~d}$ input. In $R: S S$, 2011. 